\title{
ASYMPTOTIC PROPERTIES OF KNESER TYPE SOLUTIONS FOR THIRD ORDER HALF-LINEAR NEUTRAL DIFFERENCE EQUATIONS
}

\author{
R. SRINIVASAN, C. DHARUMAN, JOHN R. GRAEF, AND E. THANDAPANI \\ Received 21 December, 2020
}

\begin{abstract}
The authors examine properties of positive solutions of the third order half-linear neutral difference equation

$$
\Delta\left(a_{n} \Delta\left(b_{n}\left(\Delta z_{n}\right)^{\alpha}\right)\right)+q_{n} y_{n+1}^{\alpha}=0,
$$

where $z_{n}=y_{n}+p_{n} y_{\sigma(n)}$. They show that the positive solutions are in fact Kneser type solutions and they provide upper and lower bounds that yield the rate of convergence to zero for such solutions. Examples are provided to illustrate the main results.
\end{abstract}

2010 Mathematics Subject Classification: 39A10

Keywords: difference equations, third order, half-linear, Kneser solutions, nonoscillation

\section{INTRODUCTION}

In this paper, we are concerned with the asymptotic properties of solutions of the third order neutral difference equation

$$
\Delta\left(a_{n} \Delta\left(b_{n}\left(\Delta z_{n}\right)^{\alpha}\right)\right)+q_{n} y_{n+1}^{\alpha}=0, \quad n \geq n_{0} \geq 0,
$$

where $z_{n}=y_{n}+p_{n} y_{\sigma(n)}, \alpha$ is the ratio of odd positive integers, and the following conditions are assumed to hold throughout:

( $\left.H_{1}\right)\left\{a_{n}\right\},\left\{b_{n}\right\}$, and $\left\{q_{n}\right\}$ are positive real sequences for all $n \geq n_{0}$;

$\left(H_{2}\right)\left\{p_{n}\right\}$ is a nonnegative real sequence with $0 \leq p_{n} \leq p<1$;

$\left(H_{3}\right)\{\sigma(n)\}$ is a sequence of integers such that $\sigma(n) \geq n$ for all $n \geq n_{0}$;

$\left(H_{4}\right) \sum_{n=n_{0}}^{\infty} \frac{1}{a_{n}}=+\infty$ and $\sum_{n=n_{0}}^{\infty} \frac{1}{b_{n}^{1 / \alpha}}=+\infty$.

By a solution of equation (1.1), we mean a nontrivial real sequence $\left\{y_{n}\right\}$ defined for all $n \geq n_{0}$ and satisfying equation (1.1). A solution $\left\{y_{n}\right\}$ of equation (1.1) is said to be oscillatory if it is neither eventually positive nor eventually negative, and nonoscillatory otherwise.

Oscillatory and asymptotic properties of solution of equation (1.1) and its special cases have been an active area of investigation in recent years; see for example $[2-5,7-19]$ and the references contained therein. The well known discrete version of 
Kiguradze's theorem [1] can be used to describe the structure of the solution space for the nonoscillatory solutions. For example, for the ordinary difference equation (see [13])

$$
\Delta^{2}\left(\left(\Delta y_{n}\right)^{\alpha}\right)+q_{n} y_{n+1}^{\alpha}=0, \quad n \geq n_{0},
$$

the set $K$ of all positive solutions has the decomposition

$$
K=K_{0} \cup K_{2}
$$

where

$$
y_{n} \in K_{0} \text { if and only if } y_{n}>0, \Delta y_{n}<0, \Delta\left(\left(\Delta y_{n}\right)^{\alpha}\right)>0, \Delta^{2}\left(\left(\Delta y_{n}\right)^{\alpha}\right)<0,
$$

and

$$
y_{n} \in K_{2} \text { if and only if } y_{n}>0, \Delta y_{n}>0, \Delta\left(\left(\Delta y_{n}\right)^{\alpha}\right)>0, \Delta^{2}\left(\left(\Delta y_{n}\right)^{\alpha}\right)<0 .
$$

A positive solution $\left\{y_{n}\right\}$ of equation (1.2) is said to be of the Kneser type if $\left\{y_{n}\right\} \in K_{0}$. In the study of the asymptotic behavior of the nonoscillatory solutions, many results were directed at obtaining criteria for $K_{2}=\varnothing$, that is, showing that the only possible nonoscillatory solutions are the Kneser type ones. We will also say that equation (1.2) has property $(A)$ if every positive solution $\left\{y_{n}\right\}$ belongs to $K_{0}$ and $\lim _{n \rightarrow \infty} y_{n}=0$.

The aim of this paper is to conduct an analogous study for equation (1.1). That is, we wish to give sufficient conditions for the nonoscillatory solutions to be of the Kneser type. In addition, we wish to obtain upper and lower bounds for such solutions. These estimates allow us to determine the rate of convergence of Kneser type solutions to zero. The results presented in this paper are new and complement those in $[4-8,11-19]$.

\section{MAin Results}

We begin with the following lemma that gives the basic properties of nonoscillatory solutions of equation (1.1). We present it for the case of positive solutions but clearly analogous statements hold for the negative solutions. As a part of this lemma, we define classes of solutions of equation (1.1) that are analogous to the sets $K_{0}$ and $K_{2}$ for equation (1.2).

Lemma 1. Assume that $\left\{y_{n}\right\}$ is a positive solution of equation (1.1). Then the corresponding sequence $\left\{z_{n}\right\}$ belongs to one of the following classes:

$$
\begin{aligned}
& S_{0}=\left\{z_{n}: z_{n}>0, \Delta z_{n}<0, \Delta\left(b_{n}\left(\Delta z_{n}\right)^{\alpha}\right)>0, \Delta^{2}\left(a_{n} \Delta\left(b_{n}\left(\Delta z_{n}\right)^{\alpha}\right)\right)<0\right\} \\
& S_{2}=\left\{z_{n}: z_{n}>0, \Delta z_{n}>0, \Delta\left(b_{n}\left(\Delta z_{n}\right)^{\alpha}\right)>0, \Delta^{2}\left(a_{n} \Delta\left(b_{n}\left(\Delta z_{n}\right)^{\alpha}\right)\right)<0\right\}
\end{aligned}
$$

eventually.

Proof. The proof is similar to that of [16, Lemma 2.1] and so we omit the details. 
We will say that the neutral difference equation (1.1) has property $(A)$, if every positive solution $\left\{y_{n}\right\}$ satisfies $\lim _{n \rightarrow \infty} y_{n}=0$ and the corresponding sequence $\left\{z_{n}\right\} \in S_{0}$.

In the following, we first present sufficient conditions for the equation (1.1) to have only Kneser type solutions. Our approach will be to first obtain some properties that solutions of equation (1.1) that belong to the class $S_{2}$ must satisfy, and use these facts to obtain sufficient conditions for the class $S_{2}$ to in fact be empty. Then we give upper and lower estimate for such solutions.

To simplify our notation we let:

$$
\begin{gathered}
A_{n}=\sum_{s=N}^{n-1} \frac{1}{a_{s}}, \quad B_{n}=\sum_{s=N}^{n-1} \frac{1}{b_{s}^{1 / \alpha},} \\
C_{n}=\sum_{s=N}^{n-1} \frac{A_{s}}{b_{s}^{1 / \alpha}}, \quad E_{n}=\left(1-p_{n} \frac{C_{\sigma(n)}}{C_{n}}\right)>0, \\
Q_{n}=\left[\frac{1}{b_{n}} \sum_{s=n}^{\infty} \frac{1}{a_{s}} \sum_{t=s}^{\infty} q_{t}\right]^{1 / \alpha}, \quad \phi_{n}=\prod_{s=N}^{n-1}\left(1+Q_{s}\right), \\
R_{n}=(1-p) \frac{\phi_{n}}{b_{n}^{1 / \alpha}}\left(\sum_{s=n}^{\infty} \frac{1}{a_{s}} \sum_{t=s}^{\infty} \frac{q_{t}}{\phi_{t+1}^{\alpha}}\right)^{1 / \alpha},
\end{gathered}
$$

where $N \geq n_{0}$.

Lemma 2. Let $\left\{y_{n}\right\}$ be a positive solution of equation (1.1) with the corresponding sequence $\left\{z_{n}\right\} \in S_{2}$ for $n \geq N \geq n_{0}$ and assume that

$$
\sum_{n=N}^{\infty} \frac{1}{a_{n}} \sum_{s=n}^{\infty} q_{s} E_{s+1}^{\alpha} B_{s+1}^{\alpha}=\infty .
$$

Then:

(i) $\left\{\frac{z_{n}}{C_{n}}\right\}$ is decreasing for all $n \geq N$;

(ii) $\left\{\frac{b_{n}^{1 / \alpha} \Delta z_{n}}{A_{n}^{1 / \alpha}}\right\}$ is decreasing for all $n \geq N$;

(iii) $\left\{\frac{z_{n}}{B_{n}}\right\}$ is increasing for all $n \geq N$.

Proof. Let $\left\{y_{n}\right\}$ be a positive solution of equation (1.1) with the corresponding sequence $\left\{z_{n}\right\} \in S_{2}$ for all $n \geq N$. Since $a_{n} \Delta\left(b_{n}\left(\Delta z_{n}\right)^{\alpha}\right)$ is decreasing, we have

$$
b_{n}\left(\Delta z_{n}\right)^{\alpha} \geq \sum_{s=N}^{n-1} \frac{a_{s} \Delta\left(b_{s}\left(\Delta z_{s}\right)^{\alpha}\right)}{a_{s}} \geq A_{n} a_{n} \Delta\left(b_{n}\left(\Delta z_{n}\right)^{\alpha}\right), \quad n \geq N .
$$

From the last inequality, we obtain

$$
\Delta\left(\frac{b_{n}\left(\Delta z_{n}\right)^{\alpha}}{A_{n}}\right)=\frac{A_{n} \Delta\left(b_{n}\left(\Delta z_{n}\right)^{\alpha}\right)-b_{n}\left(\Delta z_{n}\right)^{\alpha} \frac{1}{a_{n}}}{A_{n} A_{n+1}} \leq 0
$$


for all $n \geq N \geq n_{0}$. Thus, $\left\{\frac{b_{n}\left(\Delta z_{n}\right)^{\alpha}}{A_{n}}\right\}$ is decreasing for all $n \geq N$, so (ii) holds and

$$
z_{n} \geq \sum_{s=N}^{n-1} \frac{A_{s}^{1 / \alpha} b_{s}^{1 / \alpha} \Delta z_{s}}{A_{s}^{1 / \alpha}} \geq \frac{b_{n}^{1 / \alpha} \Delta z_{n}}{A_{n}^{1 / \alpha}} C_{n}, \quad n \geq N
$$

Hence,

$$
\Delta\left(\frac{z_{n}}{C_{n}}\right)=\frac{C_{n} \Delta z_{n}-z_{n} \frac{A_{n}^{1 / \alpha}}{b_{n}^{1 / \alpha}}}{C_{n} C_{n+1}} \leq 0,
$$

which implies that $\left\{\frac{z_{n}}{C_{n}}\right\}$ is decreasing for all $n \geq N$, so (i) holds.

Since $b_{n}^{1 / \alpha} \Delta z_{n}$ is positive and strictly increasing for any $n \geq N$, it is easy to see that for all $n \geq N_{1} \geq N$,

$$
\begin{aligned}
z_{n} & \leq z_{N_{1}}+b_{n}^{1 / \alpha} \Delta z_{n} \sum_{s=N_{1}}^{n-1} \frac{1}{b_{s}^{1 / \alpha}} \\
& =z_{N_{1}}-b_{n}^{1 / \alpha} \Delta z_{n} \sum_{s=N}^{N_{1}-1} \frac{1}{b_{s}^{1 / \alpha}}+b_{n}^{1 / \alpha} \Delta z_{n} \sum_{s=N}^{n-1} \frac{1}{b_{s}^{1 / \alpha}} .
\end{aligned}
$$

We claim that $b_{n}^{1 / \alpha} \Delta z_{n} \rightarrow \infty$ as $n \rightarrow \infty$. If this is not the case, then $b_{n}^{1 / \alpha} \Delta z_{n} \rightarrow 2 d<\infty$ as $n \rightarrow \infty$ From the definition of $z_{n}$ and using the fact that $\left\{\frac{z_{n}}{C_{n}}\right\}$ is decreasing, we have

$$
y_{n} \geq z_{n}\left(1-p_{n} \frac{C_{\sigma(n)}}{C_{n}}\right)=E_{n} z_{n} .
$$

Summing equation (1.1) from $n$ to $\infty$ and using the last inequality, we obtain

$$
\Delta\left(b_{n}\left(\Delta z_{n}\right)^{\alpha}\right) \geq \frac{1}{a_{n}} \sum_{s=n}^{\infty} q_{s} E_{s+1}^{\alpha} z_{s+1}^{\alpha} .
$$

Now $b_{n}^{1 / \alpha} \Delta z_{n} \rightarrow 2 d$ as $n \rightarrow \infty$ implies $b_{n}^{1 / \alpha} \Delta z_{n}>d$ for $n$ large enough, which in turn implies $z_{n}>d B_{n}$. Combining the last two inequalities and summing once more, we obtain

$$
(2 d)^{\alpha} \geq d^{\alpha} \sum_{n=N_{1}}^{\infty} \frac{1}{a_{n}} \sum_{s=n}^{\infty} q_{s} E_{s+1}^{\alpha} B_{s+1}^{\alpha},
$$

which contradicts (2.1). Thus, $b_{n}^{1 / \alpha} \Delta z_{n} \rightarrow \infty$ as $n \rightarrow \infty$ as we claimed.

Hence, in view of (2.3) and $\left(H_{4}\right)$, we obtain

$$
z_{n} \leq b_{n}^{1 / \alpha} \Delta z_{n} B_{n}, \quad n \geq N
$$

From the last inequality, we see that

$$
\Delta\left(\frac{z_{n}}{B_{n}}\right)=\frac{B_{n} \Delta z_{n}-\frac{z_{n}}{b_{n}^{1 / \alpha}}}{B_{n} B_{n+1}} \geq 0
$$


eventually, and we conclude that $\left\{\frac{z_{n}}{B_{n}}\right\}$ is increasing. Therefore (iii) holds and this completes the proof of the lemma.

Lemma 3. Assume that (2.1) holds and let $\left\{y_{n}\right\}$ be a positive solution of equation (1.1) with the corresponding sequence $\left\{z_{n}\right\} \in S_{2}$ for all $n \geq N$. Then

$$
E_{n} z_{n} \leq y_{n} \leq z_{n} \quad \text { for } n \geq N .
$$

Proof. From the definition of $z_{n}$, we have $z_{n} \geq y_{n}$ for all $n \geq N$. Furthermore,

$$
y_{n} \geq z_{n}-p_{n} z_{\sigma(n)} \geq E_{n} z_{n}
$$

where we have used the fact that $\left\{\frac{z_{n}}{C_{n}}\right\}$ is decreasing for all $n \geq N$. This completes the proof of the lemma.

Next, we obtain sufficient condition for all nonoscillatory solutions of (1.1) to be of the Kneser type.

Theorem 1. If condition (2.1) holds and

$$
\lim _{n \rightarrow \infty} \sup \left\{\frac{1}{A_{n+1}} \sum_{s=N}^{n} q_{s} E_{s+1}^{\alpha} C_{s+1}^{\alpha} A_{s+1}+\frac{C_{n+1}^{\alpha}}{B_{n+1}^{\alpha}} \sum_{s=n+1}^{\infty} q_{s} E_{s+1}^{\alpha} B_{s+1}^{\alpha}\right\}>1,
$$

then $S_{2}=\varnothing$.

Proof. Let $\left\{y_{n}\right\}$ be a positive solution of equation (1.1), with the corresponding sequence $\left\{z_{n}\right\} \in S_{2}$ for all $n \geq N$. Using (2.4) in equation (1.1), we obtain

$$
\Delta\left(a_{n} \Delta\left(b_{n}\left(\Delta z_{n}\right)^{\alpha}\right)\right)+q_{n} E_{n+1}^{\alpha} z_{n+1}^{\alpha} \leq 0, \quad n \geq N .
$$

Summing inequality (2.6) from $n$ to $\infty$, we have

$$
\Delta\left(b_{n}\left(\Delta z_{n}\right)^{\alpha}\right) \geq \sum_{s=N}^{n-1} \frac{1}{a_{s}}\left(\sum_{t=s}^{\infty} q_{t} E_{t+1}^{\alpha} z_{t+1}^{\alpha}\right) .
$$

From (2.7), it follows that

$$
\begin{aligned}
b_{n}\left(\Delta z_{n}\right)^{\alpha} & \geq \sum_{s=N}^{n-1} \frac{1}{a_{s}}\left(\sum_{t=s}^{\infty} q_{t} E_{t+1}^{\alpha} z_{t+1}^{\alpha}\right) \\
& =\sum_{s=N}^{n-1} \frac{1}{a_{s}} \sum_{t=s}^{n-1} q_{t} E_{t+1}^{\alpha} z_{t+1}^{\alpha}+\sum_{s=N}^{n-1} \frac{1}{a_{s}} \sum_{t=n}^{\infty} q_{t} E_{t+1}^{\alpha} z_{t+1}^{\alpha} \\
& =\sum_{s=N}^{n-1} q_{s} E_{s+1}^{\alpha} A_{s+1} z_{s+1}^{\alpha}+A_{n} \sum_{s=n}^{\infty} q_{s} E_{s+1}^{\alpha} z_{s+1}^{\alpha} .
\end{aligned}
$$

Using (2.2), in the last inequality, we obtain

$$
\frac{A_{n+1} z_{n+1}^{\alpha}}{C_{n+1}^{\alpha}} \geq \sum_{s=N}^{n} q_{s} A_{s+1} E_{s+1}^{\alpha} z_{s+1}^{\alpha}+A_{n+1} \sum_{s=n+1}^{\infty} q_{s} E_{s+1}^{\alpha} z_{s+1}^{\alpha} .
$$


In view of the monotonicity properties (i) and (iii) in Lemma 2, (2.8) yields

$$
1 \geq \frac{1}{A_{n+1}} \sum_{s=N}^{n} q_{s} A_{s+1} E_{s+1}^{\alpha} C_{s+1}^{\alpha}+\frac{C_{n+1}^{\alpha}}{B_{n+1}^{\alpha}} \sum_{s=n+1}^{\infty} q_{s} E_{s+1}^{\alpha} B_{s+1}^{\alpha} .
$$

Taking the limsup as $n \rightarrow \infty$ on both sides of the last inequality leads to a contradiction of (2.5). This completes the proof of the theorem.

Next, we wish to obtain estimates for the Kneser solutions. We begin with a lemma.

Lemma 4. Let $\left\{y_{n}\right\}$ be a positive solution of equation (1.1) with the corresponding sequence $\left\{z_{n}\right\} \in S_{0}$ for $n \geq N$. Then:

(i) $(1-p) z_{n} \leq y_{n} \leq z_{n}$ for all $n \geq N$;

(ii) $\left\{z_{n} \phi_{n}\right\}$ is increasing for all $n \geq N$.

Proof. Assume that $\left\{y_{n}\right\}$ is a positive solution of equation (1.1) with the corresponding sequence $\left\{z_{n}\right\} \in S_{0}$. Then $z_{n}$ is positive, $z_{n} \geq y_{n}$, and

$$
y_{n}=z_{n}-p_{n} y_{\sigma(n)} \geq(1-p) z_{n}, \quad n \geq N \geq n_{0},
$$

so (i) is proved.

It easy to see that $z_{n} \in S_{0}$ implies

$$
\lim _{n \rightarrow \infty} b_{n}\left(\Delta z_{n}\right)^{\alpha}=0 ;
$$

otherwise we would eventually have $\Delta z_{n}>0$ contradicting $z_{n} \in S_{0}$. Similarly,

$$
\lim _{n \rightarrow \infty} a_{n} \Delta\left(b_{n}\left(\Delta z_{n}\right)^{\alpha}\right)=0 .
$$

A summation of equation (1.1) then yields

$$
a_{n} \Delta\left(b_{n}\left(\Delta z_{n}\right)^{\alpha}\right)=\sum_{s=n}^{\infty} q_{s} y_{s+1}^{\alpha} \leq \sum_{s=n}^{\infty} q_{s} z_{s+1}^{\alpha} \leq z_{n+1}^{\alpha} \sum_{s=n}^{\infty} q_{s} .
$$

Summing once more, we obtain

$$
b_{n}\left(\Delta z_{n}\right)^{\alpha} \geq-z_{n+1}^{\alpha} \sum_{s=n}^{\infty} \frac{1}{a_{s}} \sum_{t=s}^{\infty} q_{t},
$$

or

$$
\Delta z_{n} \geq-z_{n+1} Q_{n}
$$

Hence,

$$
\Delta\left(z_{n} \phi_{n}\right)=\phi_{n} \Delta z_{n}+z_{n+1} \Delta \phi_{n} \geq z_{n+1}\left(\Delta \phi_{n}-\phi_{n} Q_{n}\right)=0
$$

since $\left\{\phi_{n}\right\}$ is a solution of the difference equation $\left(\Delta \phi_{n}-Q_{n} \phi_{n}\right)=0$. Therefore, $\left\{z_{n} \phi_{n}\right\}$ is increasing and this completes the proof of the lemma. 
Theorem 2. Assume that conditions (2.1) and (2.5) hold. If $\left\{y_{n}\right\}$ is a positive solution of equation (1.1), then there are positive constants $C_{1}$ and $C_{2}$ such that

$$
C_{1} \psi_{1}(n) \leq y_{n} \leq C_{2} \psi_{2}(n),
$$

where

$$
\psi_{1}(n)=\frac{1}{\phi_{n}} \text { and } \psi_{2}(n)=\prod_{s=N}^{n-1}\left(1-R_{s}\right) .
$$

Proof. Assume that $\left\{y_{n}\right\}$ is a positive solution of equation (1.1). Then, by Theorem $1,\left\{y_{n}\right\}$ is a Kneser type solution. From Lemma 4, we have that $\left\{z_{n} \phi_{n}\right\}$ is increasing for all $n \geq N$. Therefore,

$$
y_{n} \geq \frac{(1-p)}{\phi_{n}} z_{n} \phi_{n} \geq \frac{(1-p)}{\phi_{n}} z_{N} \phi_{N}=C_{1} \psi_{1}(n) .
$$

On the other hand, summing equation (1.1) from $n$ to $\infty$ and applying Lemma 4 (i), we have

$$
a_{n} \Delta\left(b_{n}\left(\Delta z_{n}\right)^{\alpha}\right)=\sum_{s=n}^{\infty} q_{s} y_{s+1}^{\alpha} \geq(1-p)^{\alpha} \sum_{s=n}^{\infty} q_{s} z_{s+1}^{\alpha} \geq(1-p)^{\alpha} \phi_{n+1}^{\alpha} z_{n+1}^{\alpha} \sum_{s=n}^{\infty} \frac{q_{s}}{\phi_{s+1}^{\alpha}} .
$$

Again summing the last inequality and applying Lemma 4 (ii) gives

or

$$
-b_{n}\left(\Delta z_{n}\right)^{\alpha} \geq(1-p)^{\alpha} \phi_{n}^{\alpha} z_{n}^{\alpha} \sum_{s=n}^{\infty} \frac{1}{a_{s}} \sum_{t=s}^{\infty} \frac{q_{t}}{\phi_{t+1}^{\alpha}}
$$

$$
\frac{\Delta z_{n}}{z_{n}} \leq-R_{n}, n \geq N
$$

Summing the last inequality from $N$ to $n-1$, we obtain

$$
y_{n} \leq z_{n} \leq C_{2} \psi_{2}(n) .
$$

This proves the theorem.

From Theorem 2 we deduce the following result for the half-linear difference equation (1.2).

Corollary 1. Assume that

$$
\sum_{n=N}^{\infty}\left(\sum_{s=n}^{\infty} s^{\alpha} q_{s}\right)=\infty
$$

and

$$
\lim _{n \rightarrow \infty} \sup \left\{\frac{1}{n+1} \sum_{s=N}^{n}(s+1)^{\alpha}(s+2)^{\alpha} q_{s}+(n+2)^{\alpha} \sum_{s=n+1}^{\infty}(s+1)^{\alpha} q_{s}\right\}>2^{\alpha} .
$$

Then every positive solution $\left\{y_{n}\right\}$ of equation (1.2) is decreasing and satisfies estimates of the form (2.9) with $p=0$ in $R_{n}$. 


\section{EXAMPLES}

In this section, we present two examples to illustrate the significance of our main results.

Example 1. Consider the third order difference equation

$$
\Delta^{3} y_{n}+\frac{10}{n(n+1)(n+2)} y_{n+1}=0, \quad n \geq 1 .
$$

A simple calculation shows that conditions (2.10) and (2.11) are satisfied, so by Corollary 1, positive nonoscillatory solutions of equation (3.1) are of the Kneser type and a positive solution $\left\{y_{n}\right\}$ satisfies the estimates

$$
\frac{C_{1}}{n} \leq y_{n} \leq \frac{C_{2}}{n}
$$

for some positive constants $C_{1}$ and $C_{2}$. Therefore, equation (3.1) has property (A).

Example 2. Consider the third order neutral difference equation

$$
\Delta\left(\frac{1}{n+2} \Delta^{2}\left(y_{n}+\frac{1}{2} y_{2 n}\right)\right)+\frac{3 \beta}{n(n+1)(n+2)(n+3)} y_{n+1}=0, \quad n \geq 1 .
$$

Again calculations show that conditions (2.1) and (2.5) hold if $\beta>6$. Therefore, all positive nonoscillatory solutions of equation (3.2) are of the Kneser type and Theorem 2 provides estimates for a positive solution $\left\{y_{n}\right\}$ to be of the form

$$
\frac{C_{1}}{n^{\beta}} \leq y_{n} \leq \frac{C_{2}}{n}
$$

for some positive constants $C_{1}$ and $C_{2}$. We also see that (3.2) has property (A).

\section{CONCLUSIONS}

In this paper, we give conditions for all positive solutions of a third order halflinear neutral difference equation to be of the Kneser type and we derive upper and lower bounds for them. We also wish to point out that the results obtained in Lemmas 2 and 3 are new and complement those in [4, 5, 7, 8, 11-19].

\section{REFERENCES}

[1] R. P. Agarwal, Difference Equations and Inequalities, Theory, Methods and Applications, Second Edition. New York: Dekker, 2000.

[2] R. P. Agarwal, M. Bohner, S. R. Grace, and D. O`Regan, Discrete Oscillation Theory Applications. New York: Hindawi, 2005.

[3] R. P. Agarwal, R. P. Gala, and M. A. Ragusa, "A regularity criterion in weak spaces to Boussinesq equations." Mathematics, vol. 8, no. 920, 2020.

[4] R. P. Agarwal and S. R. Grace, "Oscillation of certain third order difference equations." Comput. Math. Appl., vol. 42, pp. 379-384, 2001.

[5] R. P. Agarwal, S. R. Grace, and D. O`Regan, "On the oscillation of certain third order difference equations.” Adv. Difference Equ., vol. 2005, pp. 345-367, 2005. 
[6] B. Baculíková and J. Džurina, "Remark on properties of Kneser solutions for thirdorder neutral differential equations." Appl. Math. Letters., vol. 63, pp. 1-5, 2017, doi: 10.1016/j.aml.2016.07.005.

[7] Z. Došlá and A. Kobza, "On third order linear difference equations involving quasi-differences." Adv. Difference Equ., vol. 2006, no. 65652, pp. 1-13, 2006, doi: 10.1155/ADE/2006/65652.

[8] J. R. Graef and E. Thandapani, "Oscillatory and asymptotic behavior of solutions of third order delay difference equations.” Funkcial. Ekvac., vol. 42, pp. 355-369, 1999.

[9] C. Kome and Y. Yazlik, "The $m$-extension of Fibonacci and Lucas p-difference sequences." Filomat, vol. 33, pp. 6187-6194, 2019.

[10] M. A. Ragusa and A. Tachikawa, "Regularity for minimizers for functionals of double phase with variable exponents." Adv. Nonlinear Anal., vol. 9, pp. 710-728, 2020, doi: 10.1515/anona-20200022 .

[11] S. H. Saker, "Oscillation and asymptotic behavior of third order nonlinear neutral delay difference equations." Dynam. Systems Appl., vol. 15, pp. 549-567, 2006.

[12] S. H. Saker, "New oscillation criteria for third order nonlinear neutral difference equations." Math. Slovaca, vol. 61, pp. 579-600, 2011, doi: 10.2478/s12175-011-0030-5.

[13] S. H. Saker, J. O. Alzabut, and A. Mukheimer, "On the oscillatory behavior for a certain class of third order nonlinear delay difference equations." Electron. J. Qual. Theory Differ. Equ., vol. 2010, no. 67, pp. 1-16, 2010, doi: 10.14232/ejqtde.2010.1.67.

[14] B. Smith, "Oscillatory and asymptotic behavior in certain third order difference equations." Rocky Mountain J. Math., vol. 17, pp. 597-606, 1987, doi: 10.1216/RMJ-1987-17-3-597.

[15] A. A. Sobilo and A. Drozdowicz, "Asymptotic behavior of solutions of third order nonlinear difference equations of neutral type." Math. Bohem., vol. 133, pp. 247-258, 2008.

[16] E. Thandapani and K. Mahalingam, "Oscillatory properties of third order neutral delay difference equations.” Demonstratio Math., vol. 35, pp. 325-337, 2002.

[17] E. Thandapani and S. Selvarangam, "Oscillation results for third order half-linear neutral difference equations," Bull. Math. Anal. Appl., vol. 4, pp. 91-102, 2012.

[18] E. Thandapani and S. Selvarangam, "Oscillation of third order half-linear neutral difference equations." Math. Bohem., vol. 138, pp. 87-104, 2013.

[19] E. Thandapani, M. Vijaya, and T. Li, "On the oscillation of third order half-linear neutral type difference equations.” Electron. J. Qual. Theory Differ. Equ., vol. 2011, no. 76, pp. 1-13, 2011, doi: 10.14232/ejqtde.2011.1.76.

Authors' addresses

R. Srinivasan

SRM University, Department of Mathematics, Ramapuram Campus, Chennai 600 089, India

E-mail address: srinimaths1986@gmail.com

C. Dharuman

SRM University, Department of Mathematics, Ramapuram Campus, Chennai 600 089, India

E-mail address: cdharuman55egmail.com

John R. Graef

(Corresponding author) University of Tennessee at Chattanooga, Department of Mathematics, Chattanooga, TN 37403, USA

E-mail address: John-Graef@utc.edu 


\section{E. Thandapani}

University of Madras, Ramanujan Inst. for Advanced Study in Math., Chennai 600 005, India

E-mail address: ethandapani@yahoo.co.in 